\title{
La terapia cognitivo conductual mejora los síntomas de insomnio asociado a condiciones psiquiátricas o médicas
}

\author{
Cognitive behavioral therapy improves symptoms of insomnia associated with psychiatric or medical conditions
}

\section{Objetivos}

Evaluar la eficacia de la terapia cognitivo conductual para el insomnio (TCC-I) en pacientes con insomnio asociado a enfermedades médicas o psiquiátricas a través de diferentes variables, como la remisión del insomnio, la eficiencia, la latencia de inicio de sueño, el tiempo de vigilia después de iniciado el sueño, el tiempo total de sueño, la calidad de sueño y sus efectos sobre la condición comórbida.

Fuentes, selección de estudios y extracción de datos Se realizaron búsquedas en Pubmed, PsycINFO y la Biblioteca

Tabla 1. Eficacia de la terapia cognitivo conductual para insomnio
Wu JQ y col. JAMA Interm Med. 2015;175(9):1461-1472

Cochrane, seguida de una búsqueda manual de las referencias de las revisiones sistemáticas hasta junio 2014. Se seleccionaron, de forma independiente, ensayos clínicos controlados aleatorizados que compararan TCC-I con controles sin este tipo de terapia, en pacientes mayores de 18 años con criterios de insomnio y comorbilidad asociada. Los estudios debían reportar datos que permitieran calcular el tamaño del efecto, que fueran extraídos de diarios de sueño u otras mediciones auto-reportadas.

Resultados principales

Se incluyeron 37 estudios. Los resultados se resumen en la tabla 1.

\begin{tabular}{|c|c|c|c|}
\hline Variables & Medida de resultado & Estimación (IC 95\%) & P-valor \\
\hline Remisión de insomnio (OR) & Odds Ratio & $3,28(2,30$ a 4,68$)$ & $<0,001$ \\
\hline Eficiencia de sueño & \multirow[t]{8}{*}{ Hedges $g^{a}$} & $0,91(0,74$ a 1,08$)$ & $<0,001$ \\
\hline Latencia de inicio de sueño & & $0,80(0,60$ a 1,00$)$ & $<0,001$ \\
\hline Tiempo despierto tras iniciado el sueño & & $0,68(0,60$ a 0,98$)$ & $<0,001$ \\
\hline Tiempo total de sueño & & $0,19(0,06$ a 0,31$)$ & 0,003 \\
\hline Calidad de sueño & & $0,84(0,69$ a 1,00$)$ & $<0,001$ \\
\hline Comorbilidad & & $0,39(0,25$ a 0,53$)$ & $<0,001$ \\
\hline Comorbilidad psiquiátrica & & $0,76(0,46$ a 1,05$)$ & $<0,001$ \\
\hline Comorbilidad medica & & $0,20(0,09$ a 0,30$)$ & $<0,001$ \\
\hline
\end{tabular}

a Hedges g: determina tamaños de efecto (en variables no paramétricas) pre y postratamiento, considerando 0,2 un efecto pequeño, 0,5 mediano y 0,8 grande.

\section{Conclusión}

La TCC-I puede mejorar los síntomas de insomnio y la calidad de sueño en las personas con insomnio asociado a comorbilidades médicas y psiquiátricas, encontrándose un efecto moderado a grande en la mayoría de los parámetros evaluados salvo en el tiempo total de sueño. Además, puede afectar los síntomas de la condición comórbida con mayor efecto sobre una condición psiquiátrica.

Conflictos de interés de los autores: No referido.

\section{Comentario}

La TCC-I es una intervención psicológica multicomponente que consiste en control de estímulos, restricción del tiempo en cama, técnicas de relajación, reestructuración cognitiva e intención paradójica. Dura de 4 a 10 semanas y se realiza en varias sesiones ${ }^{1}$. En el manejo inicial del insomnio se recomienda al menos una intervención psicológica y usar el tratamiento farmacológico como coadyuvante en caso de falla. Esta terapia demostró ser efectiva aumentando la eficiencia de sueño disminuyendo la latencia de inicio de sueño y mejorando la calidad de vida en comparación con los controles, y ayuda a disminuir el uso de fármacos ${ }^{1}$ En meta-análisis previos se había determinado su eficacia en el tratamiento del insomnio primario, pero en la práctica clínica la mayoría de los pacientes consultan por insomnio como síntoma de una patología subyacente, problema que es abordado por este estudio. Se ha descripto que tratar la enfermedad de base no es suficiente para mejorar los síntomas de insomnio asociado a comorbilidades; es necesario hacer un abordaje terapéutico independiente, ya que este mejora los síntomas de insomnio y, a su vez, influye positivamente en la enfermedad de manera bidireccional $^{2-7}$. Esta revisión sistemática y meta-análisis demuestra que la TCC-I es eficaz en comparación con otras alternativas terapéuticas en este tipo de pacientes, ya que mejora todos los parámetros del sueño con excepción del tiempo total de sueño, aumenta la remisión del insomnio y mejora los síntomas de la enfermedad de base, sobre todo en comorbilidades psiquiátricas. Entre las debilidades de este estudio se destacan que todos los ensayos revisados incluían un número pequeño de pacientes, todas las mediciones eran subjetivas, solo 10 estudios incluían métodos objetivos como la actigrafía y la polisomnografía, por lo que los resultados sobre los efectos objetivos de la TCC-I aún son inconcluyentes. Por otro lado, en los ensayos no estaban representadas la totalidad de las enfermedades psiquiátricas y médicas, y los desenlaces medidos de las condiciones comórbidas eran muy heterogéneos. Adicionalmente, el efecto a largo plazo (3 a 12 meses) fue evaluado en pocos estudios.

\section{Conclusiones de la comentadora}

Todo médico debería categorizar los síntomas de insomnio referidos por los pacientes y tratarlo en paralelo a su trastorno primario, siendo recomendado abordarlo con TCC-I. Si bien la limitante en nuestro medio es la disponibilidad, es necesario tener en cuenta que no solo puede ser administrada por especialistas como psiquiatras y psicólogos, sino también por enfermeros capacitados, y que se puede adaptar la duración de la terapia, el número de sesiones, así como su enfoque grupal o individual, para así lograr un mayor alcance de este recurso terapéutico.

María Jimena Madrazo [ Hospital Italiano de Buenos Aires. jimemadrazo@ gmail.com]

Madrazo MJ. La terapia cognitivo conductual mejora los síntomas de insomnio asociado a condiciones psiquiátricas o médicas. Evid Act Pract Ambul 2018;21(2):53. Comentado de: Wu JQ, y col. Cognitive Behavioral Therapy for Insomnia Comorbid With Psychiatric and Medical Conditions: A Meta-analysis. JAMA Intern Med. 2015;175(9):1461-72. doi: 10.1001/jamainternmed.2015.3006. PubMed PMID: 26147487.

Referencias

1. Guía de Práctica Clínica para el Manejo de Pacientes con Insomnio en Atención Primaria. mayo de 2010. Ministerio de Ciencia e Innovación. 2. Morin CM, y col. Nonpharmacological interventions for insomnia: a meta-analysis of treatment efficacy. Am J Psychiatry. 1994;151(8):1172-1180.

3. Murtagh DRR, Greenwood KM. Identifying effective psychological treatments for insomnia: a meta-analysis. J Consult Clin Psychol. 1995;63(1):79-89. 4. rwin MR, y col. Comparative meta-analysis of behavioral interventions for insomnia and their efficacy in middle-aged adults and in older adults $55+$ years of age. Health Psychol. 2006;25(1):3-14

5. Okajima I, y col. A meta-analysis on the treatment effectiveness of cognitive behavioral therapy for primary insomnia. Sleep Biol Rhythms. 2011;9(1):24-34.

6. Koffel EA, y col. A meta-analysis of group cognitive behavioral therapy for insomnia. Sleep Med Rev. 2015;19(2):6-16.

7. Smith MT, y col. Comparative meta-analysis of pharmacotherapy and behavior therapy for persistent insomnia. Am J Psychiatry. 2002;159(1):5-11. 